\title{
ATIVIDADE DE BIOEXTRATOS NO DESENVOLVIMENTO DE PHOMOPSIS PHASEOLI VAR. SOJAE, FUSARIUM SP. E NO TRATAMENTO DE SEMENTES DE SOJA
}

\author{
Luiz Carlos Pascuali* \\ José Wilson Pires Carvalho** \\ Aniele Arvani Souza** \\ Larissa Regina Ballerini Gonçales **** \\ Armando da Silva Filho*****
}

RESUMO: As doenças de plantas são responsáveis por perdas econômicas em todos os cultivos, afetam as plantas no campo e as sementes durante o armazenamento. Diferentes técnicas de controle de patógenos são utilizadas para minimizar impactos negativos, entre elas têm-se usado bioextratos, os quais se apresentam como alternativas desejáveis comparados ao controle químico convencional. O presente estudo objetivou avaliar a atividade antifúngica in vitro e in vivo de extratos de pinhão manso (Jatropha curcas L.), cipreste (Cupressus sp.) e tiririca (Cyperus rotundus L.), alho (Allium sativum L.) cebola (Allium cepa L.) e gengibre (Zingiber officinalis L.) com diferentes processos de elaboração, contra Phomopsis phaseoli var. sojae, Fusarium sp., e a influência na germinação e no vigor de sementes de soja. Os bioextratos foram preparados utilizando-se $40 \mathrm{~g}$ de plantas (in natura e seco a $55 \pm 2{ }^{\circ} \mathrm{C}$ ) e solução água-metanol (1:3). Os extratos, após filtrados e evaporado o álcool, foram divididos em duas frações, uma delas foi autoclavada e ambas aplicadas nos isolados de culturas puras e diretamente nas sementes de soja. A secagem promoveu melhora na eficiência dos bioextratos de alho, cebola e gengibre no controle in vitro de Phomopsis phaseoli var. sojae enquanto que os de cipestre e pinhão manso foram menos efetivos. A autoclavagem resultou em perda da capacidade fungitóxica do bioextrato de alho, enquanto no de cebola

* Engenheiro Agrônomo: Doutorado em Ciência e Tecnologia de Sementes e Docente Doutor da Universidade do Estado de Mato Grosso (UNEMAT) no curso de Engenharia de Produção Agroindustrial, Brasil.

** Docente Doutor da Universidade do Estado de Mato Grosso (UNEMAT) no curso de Engenharia de Alimentos e Produção Agroindustrial, Brasil. E-mail: jwilsonc@unemat.br

*** Acadêmica da Universidade do Estado de Mato Grosso (UNEMAT) no curso de Engenharia de Produção Agroindustrial, Brasil.

**** Acadêmica da Universidade do Estado de Mato Grosso (UNEMAT) no curso de Engenharia de Produção Agroindustrial, Brasil.

***** Graduado em Ciência da Computação: Mestrado em Física Ambiental e Docente Mestre da Universidade do Estado de Mato Grosso (UNEMAT) no curso de Ciência da Computação, Brasil. 
afetou negativamente o vigor das sementes a $10 \% \mathrm{v} / \mathrm{v}$. O extrato de alho seco não autoclavado controlou eficientemente o desenvolvimento de Phomopsis phaseoli var. sojae in vitro. Os demais bioextratos não apresentaram eficiência significativa no controle dos patógenos. Todos os bioextratos conferiram à semente de soja índice de germinação inferior quando comparado ao tratamento com Carbendazin + Tiran. $\mathrm{O}$ bioextrato de cebola, gengibre ambos in natura não autoclavado e de tiririca desidratada não autoclavado melhoraram significativamente o percentual de plântulas normais, comparado à testemunha. Entretanto, os bioextratos não diminuíram a contaminação das sementes por Fusarium spp. e Phomopsis phaseoli var. sojae. Portanto, o processo de autoclavagem é uma etapa importante podendo afetar de maneira diferente a eficiência dos bioextratos estudados, assim como o processo de secagem da matéria vegetal antes da preparação dos bioextratos.

PALAVRAS-CHAVE: Alho; Cebola; Controle fúngico; Extração hidroalcoólica; Meio ambiente.

\section{BIOEXTRACT ACTIVITY IN THE DEVELOPMENT OF PHOMOPSIS PHASEOLI VAR. SOJAE, FUSARIUM SP. AND IN THE TREATMENT OF SOYBEAN SEEDS}

ABSTRACT: Plant diseases cause high economic liabilities. They affect plants on the field and seeds during storage. Different techniques for the control of pathogens are employed to minimize negative impacts. Bio-extracts are alternatives when compared to conventional chemical control. Current analysis evaluates in vitro and in vivo anti-fungus activity of extracts of jatropha (Jatropha curcas L.), cypress (Cupressus sp.), nutsedge (Cyperus rotundus L.), garlic (Allium sativum L.), onion (Allium cepa L.) and ginger (Zingiber officinalis L.), in different processes, against Phomopsis phaseoli var. sojae, Fusarium sp., and their influence on germination and vigor of soybean seeds. Bio-extracts were prepared with $40 \mathrm{~g}$ of the plants (in natura and dried at $55 \pm 2{ }^{\circ} \mathrm{C}$ ) and a water-methanol solution (1:3). After filtering and the evaporation of the alcohol, the extracts were divided into two fractions: one was autoclaved and both were applied to isolates of pure cultures directly on the soybean seeds. Drying improved the efficiency of garlic, onion and ginger bio-extracts in in vitro control of Phomopsis phaseoli var. sojae, whereas cypress and jatropha bio-extracts were less efficient. Autoclave caused the loss of the fungus-toxic capacity of garlic, whereas it affected negatively onion seed vigor at $10 \% \mathrm{v} / \mathrm{v}$. Non-autoclaved dry garlic extract controlled efficaciously the in vitro development of Phomopsis phaseoli var. sojae. The other bio-extracts failed to have any significant efficiency on pathogen control. All bio-extracts provided soybean seeds a lower germination rate 
when compared to treatment with Carbendazin + Tiran. Non-autoclaved onion and ginger bio-extracts and dehydrated nutsedge significantly improved the percentage of normal seedlings when compared to control. However, bio-extracts failed to decrease contamination rate of seeds for Fusarium spp. and Phomopsis phaseoli var. sojae. Auto-clave is, therefore, an important stage and affects differently the efficiency of the bio-extracts analyzed and the drying process of the vegetal material prior to the preparation of the bio-extracts.

KEY WORDS: Garlic; Onion; Fungus control; Hydro-alcohol extract; Environment.

\section{INTRODUÇÃO}

As doenças de plantas são responsáveis por consideráveis perdas, em especial para as culturas de importância econômica. Estas doenças podem ocorrer, no campo, em qualquer fase do ciclo da planta, como também em pós-colheita e durante o armazenamento (FLÁVIO et al., 2014; GODOY et al., 2014). Para minimizar os efeitos negativos dos microrganismos, vários métodos físicos, químicos ou biológicos vêm sendo empregados (MAIA et al., 2011; MEDEIROS et al., 2013b); SANTOS et al., 2013; GOUVEA et al., 2011; FLÁVIO et al., 2014). Os métodos físicos e biológicos constituem-se alternativas viáveis e desejáveis, em relação ao químico convencional. O emprego dos denominados fungicidas naturais aparece como mais uma opção ao uso dos fungicidas sintéticos, em termos de eficiência de controle de fungos (FRIAS et al., 2010; BUENO JÚNIOR et al., 2012; MEDEIROS et al., 2013a; SOUZA; CARDOSO, 2013).

Cruz et al. (1999a) utilizaram a fumigação com água aromatizada dos seguintes gêneros de plantas medicinais Tetradenia, Rosmarinus, Zingiber, Ocimum, Artemísia, Achillea, Mentha, Baccharis, Ruta, Laurus e Eucalyptus, em sementes de soja, o que resultou no aumento de $42 \%$ das plântulas sadias. Os autores observaram também que todos os tratamentos reduziram o percentual de sementes infectadas por Penicillium e estes mesmos gêneros de plantas foram responsáveis por aumentos de até 56,25\% de plântulas normais (CRUZ et al., 1999b).

Estudos realizados por Flávio et al. (2014) mostram que bioextrato aquoso de canela (Cinnamomum zeylanicum), concentração de 10-30\% v/v, foi efetivo 
na inibição de crescimento de fungos como Penicillium, Aspergillus, Rhizopus, Alternaria, Curvularia, Fusarium sp. em semente de sorgo, e esta inibição é diretamente proporcional às concentrações usadas. Além disso, Aquino et al., (2010) demonstraram que os bioextratos de alecrim-pimenta (Lippia sidoides), capim-santo (Cymbopogon citratus), alfavaca-cravo (Ocimum gratissimum), cravoda-índia (Syzygium aromaticum), pimenta do reino (Syzygium aromaticum), também possuem efeito inibitório sobre os fungos Aspergillus sp. e Penicillium sp. em sementes de girassol.

Hillen et al. (2012) utilizaram óleos essenciais de candeia (Eremanthus erythropappus), palmarrosa (Cymbopogon martinii) e alecrim (Rosmarinus officinalis), para o tratamento de sementes de milho, soja e feijão com diferentes fungos patogênicos, e verificaram que o alecrim reduziu completamente a ocorrência de patógenos, no entanto, inibiu também a germinação. A candeia controlou a ocorrência dos patógenos, a partir de formulações a $1 \%$ de óleo. O óleo essencial de palmarrosa mostrou-se eficiente na inibição dos fungos Alternaria carthami, Alternaria sp. e Rbizoctonia solani, entretanto, a percentagem de germinação mostrou-se inversamente proporcional ao aumento da concentração aplicada.

Bueno Júnior et al. (2012) observaram que o bioextrato de Momordica charantia L é eficiente no controle de Sclerotinia sclerotiorum em semente de feijão, contudo, ocorreu redução na porcentagem de germinação das sementes tratadas.

A suspensão aquosa de óleo essencial e água aromatizada de manjericão (Ocimum basilicum), capim limão (Cymbopogon citratus (DC) Stapt) e eucalipto (Eucalyptus citriodora), aplicados em sementes de soja, controlaram Penicillium sp. e Aspergillus sp. (INQUE et al., 1998). Os autores avaliaram ainda sementes infectadas com Curvularia sp., Cercospora kikuchii, Fusarium semitectum, Aspergillus sp. e Phomopsis sp., tratadas com suspensão aquosa de óleos essenciais de losna, capim limão, eucalipto e manjericão, e obtiveram redução da incidência em todos os tratamentos; porém, observaram efeito alelopático sobre as sementes, que resultou na redução total das plântulas normais. Além disso, Inácio et al. (2009), trabalhando com Phomopsis phaseoli var. sojae, Fusarium sp. e Macrophomina phaseolina, verificaram que a aplicação dos óleos essenciais na concentração de 5.000 ppm de erva-cidreira (Melissa officinalis), capim-cidreira (Cymbopogon citratus), citronela 
(Cymbopogon winterianus) e canela (Cinnamomum zeylanicum) e o tratamento padrão com Carbendazim + Thiram (5.000 ppm em meio de cultura BDA), inibiram completamente o crescimento micelial dos patógenos.

Conforme trabalho realizado por Pastro et al. (2012), bioextratos hidroalcoólicos de cravo medicinal e cravo-da-índia (Syzygium aromaticum (L.) Merr. \& Perry) inibiram completamente o desenvolvimento de Phomopsis phaseoli var. sojae in vitro. Dentre os bioextratos testados pelos autores destacaram-se ainda o alho (Allium sativum) (branco e roxo), alecrim (Rosmarinus officinalis), pimenta do reino (Piper nigrum L), canela (Cinnamomum zeylanicum) e juá (Solanum palinacanthum Dunal) e apresentaram reduções significativas no desenvolvimento das colônias. Paula et al. (2009) observaram que os bioextratos hidroetanólicos de alho (roxo e branco) e juá não interferem na germinação de sementes de soja.

O bioextrato hidrometanólico de gengibre (Zingiber officinale Roscoe) reduziu o desenvolvimento de Fusarium spp. in vitro e o bioextrato de tiririca (Cyperus rotundus L) aumentou o índice de germinação de sementes de soja (AZEVEDO; PASCUALI, 2008). Souza et al. (2010) verificaram efeito do bioextrato hidrometanólicos de alho na inibição de Aspergillus sp. em sementes de ingá (Inga edulis) apresentando maior eficiência de inibição do fungo em concentração de bioextrato a partir de $50 \% \mathrm{v} / \mathrm{v}$.

Portanto, a aplicação de óleos essenciais e bioextratos no controle de patógenos em sementes e frutos vêm sendo objeto de estudo em vários trabalhos. Diante do exposto, o presente estudo objetivou avaliar o efeito do processo de elaboração dos bioextratos de pinhão manso (Jatropha curcas L.), cipreste (Cupressus sp.), tiririca (Cyperus rotundus L.), bulbos de alho (Allium sativum L.), cebola (Allium cepa L.) e gengibre (Zingiber officinalis L.) e a influência destes no controle in vitro e in vivo de Phomopsis phaseoli var. sojae, e, Fusarium sp., bem como o efeito sobre a germinação e vigor de sementes de soja.

\section{MATERIAL E MÉTODOS}

O presente estudo foi desenvolvido nos Laboratórios de Microbiologia e de Química, do Curso de Engenharia de Alimentos, localizado no Campus Universitário "Deputado Estadual Renê Barbour", no município de Barra do Bugres/ 
MT, pertencente à Universidade do Estado de Mato Grosso - UNEMAT.

A coleta de pinhão manso (Jatropha curcas L.), cipreste (Cupressus sp.) e tiririca (Cyperus rotundus L.) foi realizada na fazenda Arco-Íris (Barra do Bugres/ MT) e os bulbos de alho (Allium sativum L.) e cebola (Allium cepa L.) e os rizomas de gengibre (Zingiber officinalis L.) adquiridos no comércio local.

As sementes de soja foram obtidas junto aos produtores locais em áreas com colheita atrasada (áreas descartadas para produção de sementes) a fim de obter altos índices de contaminação com os patógenos em estudo.

Para obtenção dos bioextratos utilizaram-se folhas de pinhão manso, cipreste, e tiririca, bulbos de alho e cebola e rizomas de gengibre. Na preparação dos bioextratos os materiais foram fracionados em duas porções para a extração dos produtos "in natura" armazenada sobre refrigeração e a outra parte seca em estufa com circulação forçada de ar regulada na temperatura de $55 \pm 2^{\circ} \mathrm{C}$, conforme Falkenberg et al. (2000), para preparar a extração com os materiais desidratados.

Após a secagem das matérias-primas, todos os tratamentos foram preparados para a extração utilizando-se $40 \mathrm{~g}$ de material vegetal in natura e seca, $20 \mathrm{~mL}$ de água destilada e $60 \mathrm{~mL}$ de metanol, em frascos com capacidade para $200 \mathrm{~mL}$. Os frascos foram acondicionados à temperatura de $20^{\circ} \mathrm{C}$ em agitação constante durante cinco dias. Posteriormente, realizou-se a filtragem dos bioextratos em papel filtro (INLAB tipo 10) e imediatamente em membrana filtrante de porosidade $0,45 \mu \mathrm{m}$. O bioextrato filtrado foi submetido à evaporação em vácuo, para remoção do metanol, e reconstituído para $20 \mathrm{~mL}$ com água destilada e uma metade dos bioextratos foi autoclavada.

Na aplicação dos bioextratos foi usado $1 \mathrm{~mL}$ de bioextrato vegetal para cada $10 \mathrm{~mL}$ (concentração 10\% v/v) de meio de cultura BDA fundente (aproximadamente $45^{\circ} \mathrm{C}$ ), e para o fungicida Derosal Plus ${ }^{\circledR}$ (Carbendazin + Tiran), utilizaram-se 0,2 $\mathrm{mL}$ para cada $10 \mathrm{~mL}$ de meio de cultura BDA fundente (aproximadamente $45^{\circ} \mathrm{C}$ ). No tratamento das sementes realizou-se a aplicação de $3 \mathrm{~mL}$ de bioextrato para 100 gramas de sementes, mantidas em bandejas para redução do teor de umidade para $13 \%$ aproximadamente. Para o fungicida, utilizou-se a dose recomendada $(200 \mathrm{~mL}$ $100 \mathrm{~kg}^{-1}$ de sementes). Sendo usada para testemunha uma amostra de mesmo peso na qual se inocularam $3 \mathrm{~mL}$ de água destilada.

Os patógenos foram isolados das sementes de soja em meio de cultura BDA 
e replicados até a purificação e então cultivados em culturas (monospórica) puras.

A inoculação das placas foi realizada a partir de colônias puras crescidas na temperatura de $20 \pm 2^{\circ} \mathrm{C}$ com regime de 12 horas de luz, por sete dias em placas com meio de cultura BDA; foram retirados discos de $8 \mathrm{~mm}$ de diâmetro, sendo estes transferidos para o centro de cada uma das placas que compunham os diferentes tratamentos. Foram utilizadas quatro placas por tratamento.

Após a adição dos discos procedeu-se a incubação das placas em câmara apropriada em temperatura de $20 \pm 2{ }^{\circ} \mathrm{C}$ com regime de 12 horas de luz, por sete dias. O crescimento das colônias foi acompanhado por meio de medições de seus diâmetros, mensurados aos três, cinco e sete dias, em duas direções, formando um ângulo de $90^{\circ}$, calculando-se então a média entre duas medições para obtenção do diâmetro de cada colônia.

$O$ teste de germinação foi realizado com 400 sementes de soja para cada tratamento empregando-se como substrato papel "Germitest", esterilizado e umedecido com água destilada, na proporção de 2,5 vezes o peso do papel de acordo com o descrito nas Regras para Análises de Sementes - RAS para o substrato papel (BRASIL, 2009). Os rolos com 50 sementes foram colocados em germinador do tipo "Mangelsdorf" regulado na temperatura constante de $25^{\circ} \mathrm{C}$. As contagens foram realizadas nos quinto e no oitavo dias, e o resultado expresso em percentagem de plântulas normais.

No teste de envelhecimento acelerado as sementes foram acondicionadas em caixas plásticas tipo "gerbox" $(11 \times 11 \times 3 \mathrm{~cm})$ contendo $40 \mathrm{~mL}$ de água destilada, sendo distribuídas $42 \mathrm{~g}$ de sementes em camada uniforme e única, sobre a tela que isola as mesmas do contato direto com a água. As caixas foram acondicionadas em câmara tipo "Biological Organism Development" (BOD) reguladas na temperatura constante de $41^{\circ} \mathrm{C}$ durante 48 horas (MARCOS FILHO, 1999). Após este período realizou-se o teste de germinação conforme metodologia descrita por Brasil (2009). As contagens foram realizadas no quinto dia e o resultado expresso em percentagem de plântulas normais.

A sanidade das sementes foi avaliada por teste do papel filtro com 200 sementes, distribuídas em oito repetições de 25 sementes e colocadas sobre duas folhas de papel mata-borrão previamente umedecidas com água destilada, em caixas 
plásticas tipo "gerbox" e incubadas na temperatura de $20 \pm 2^{\circ} \mathrm{C}$, com regime de 12 horas de luz, por sete dias. Após esse período as sementes foram individualmente analisadas em lupa estereoscópica e quando necessário realizado o preparado de lâminas para verificação em microscópio identificando-se os fungos a elas associados em nível de gênero (Fusarium spp.) e de espécie ( $P$. phaseoli var. sojae). E os resultados expressos em percentagem de sementes infestadas, para cada patógeno.

O delineamento experimental utilizado foi o inteiramente casualizado com quatro repetições por tratamento. O efeito da secagem e da autoclavagem na eficácia dos bioextratos foi comparado pelo teste "F", e a eficácia dos bioextratos foi comparada por meio do teste de Dunnett a 5\% com duas testemunhas (com e sem tratamento químico), (SAS, 1999).

\section{RESULTADOS E DISCUSSÃO}

O resultado da análise Anova (Tabela 01) mostra as influências da secagem e autoclavagem dos bioextratos de plantas sobre o desenvolvimento de Fusarium spp., Phomopsis phaseoli var. sojae, teste de germinação, envelhecimento acelerado (EA) e sanidade de sementes de soja. 


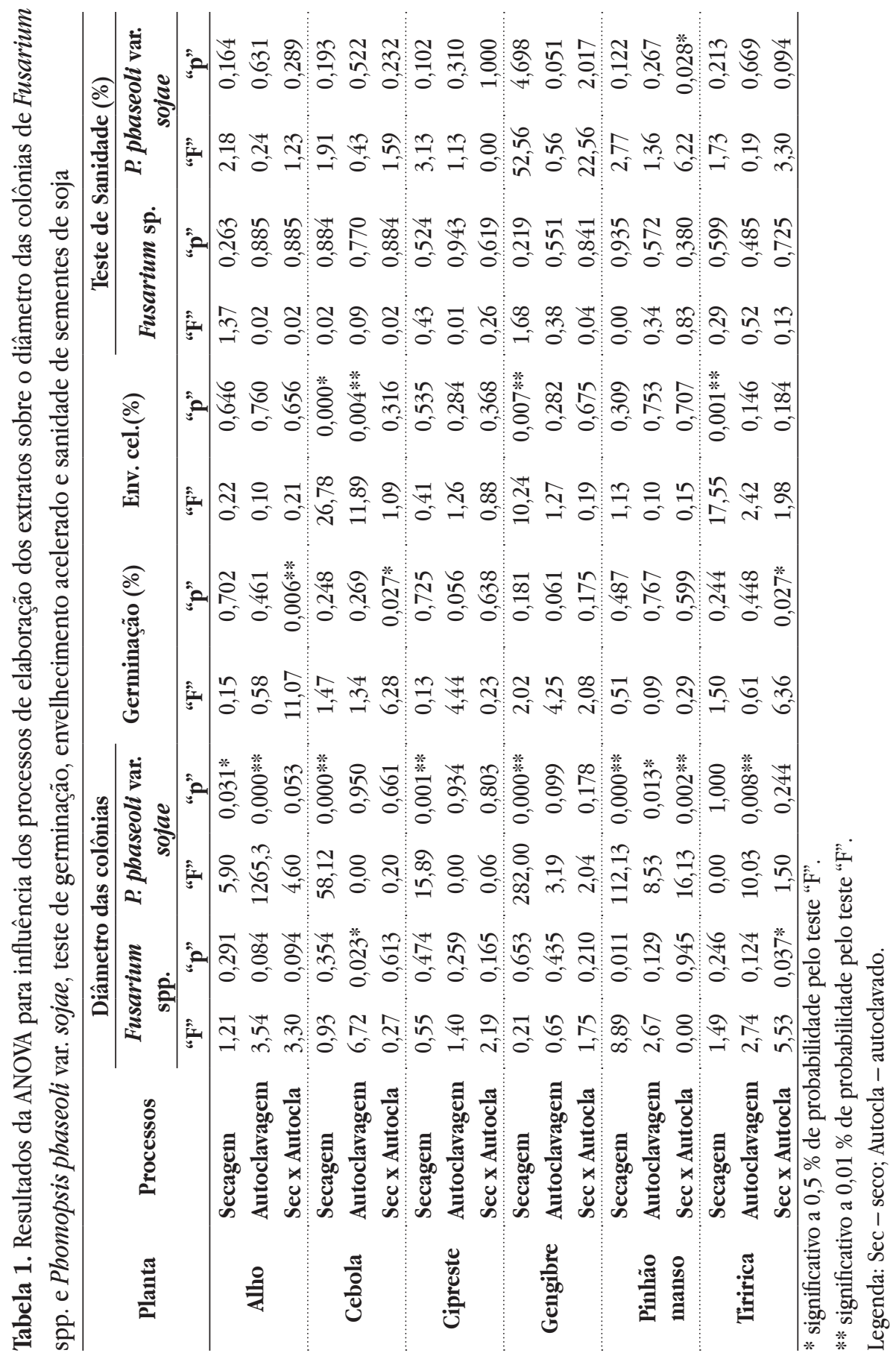


Com base nos resultados do teste "F", verificou-se que a secagem das plantas levou a melhora significativa da eficiência dos bioextratos de alho, cebola e gengibre para controle de in vitro de $P$. phaseoli var. sojae, e do bioextrato de tiririca sobre o índice de plântulas normais e envelhecimento acelerado. A melhora pode estar associada, provavelmente, à maior concentração das substâncias bioativas na planta desidratada. A secagem reduziu significativamente os efeitos dos bioextratos de cipreste e pinhão manso sobre $P$. phaseoli var. sojae, in vitro. Isso se deve, provavelmente, à degradação de moléculas levando à inativação da(s) substância(s) bioativa(s) e/ou a produção de novas moléculas fitotóxicas, como demonstrado pelo efeito negativo dos bioextratos de cebola e gengibre, sobre o percentual de plântulas normais do teste de EA. De acordo com a literatura, o alho (Allium sativum L.) possui alicina, o pinhão manso (Jatropha curcas L.) flavonoides e cianógenos, o gengibre Zingiber officinalis L.) geranial e neral, cipreste (Cupressus sp.) alfapineno, mirceno, cedrol, cedrino, terpinoleno, limoneno, cebola (Allium cepa L.) flavonoides e antocianinas, que são responsáveis pela atividade antimicrobiana destes bioextratos (ANDRADE, et al., 2012; KUMAR, et al., 2010; LEAL; AGRA, 2005; NUUTILA, et al., 2003; COWAN, 1999).

$O$ processo de autoclavagem reduziu significativamente a eficiência dos bioextratos de alho e tiririca para o controle in vitro de P. phaseoli var. sojae (Tabela 01), concordando com os resultados obtidos por Venturoso et al. (2009), que indicam a perda de eficiência do bioextrato de alho quando submetido à autoclavagem. Ainda, o bioextrato de cebola autoclavado apresentou significativa perda de eficiência sobre o controle de Fusarium spp., além de afetar negativamente o percentual de plântulas normais do teste de envelhecimento acelerado (EA). Entretanto, o bioextrato de pinhão manso teve sua eficiência melhorada com a autoclavagem para este patógeno. A diminuição da eficiência após autoclavagem pode ser explicada pela degradação de moléculas bioativas pelo calor aplicado no processo de autoclavagem a $105^{\circ} \mathrm{C}$ como provavelmente ocorre com a secagem das plantas. Os demais bioextratos não influenciaram as variáveis analisadas (Tabela 01), com exceção para a interação (secagem $\mathrm{x}$ autoclavagem) de alho, cebola e tiririca que foi significativa para o teste de germinação, pinhão manso tanto para o diâmetro das colônias bem como para ao percentual de contaminação das sementes, e de tiririca para o diâmetro das colônias de Fusarium spp. 
A obtenção dos bioextratos com as plantas na forma in natura e/ou secas, submetidos ou não à autoclavagem, permite verificar os efeitos deste processo sobre o desempenho dos bioextratos estudados. Isto pode ser confirmado com a perda de eficiência do bioextrato de cebola quando submetido à secagem e/ou autoclavado. Por sua vez, o bioextrato de gengibre mostrou melhor desempenho no controle de $P$. phaseoli var. sojae quando obtido da matéria seca, independente da autoclavagem. A análise destes resultados fornece informações úteis para a escolha do(s) processo(s) mais adequado para cada planta na elaboração dos bioextratos para aplicação no controle fúngico.

O bioextrato de alho submetido à secagem não autoclavado (Tabela 02) apresentou resultados similares à testemunha com tratamento químico (Carbendazin + Tiran), impedindo completamente o desenvolvimento de $P$. phaseoli var. sojae. Os resultados fornecidos por todos os demais processos e bioextratos diferem significativamente da testemunha. Resultados semelhantes foram obtidos por Nascimento; Nery; Rodrigues, (2006), usando bioextratos concentrados de alho (Allium sativum L.) e angico (Anadenanthera peregrina L. Speng) com o controle de Colletotrichum gloeosporioides, agente causador da podridão de frutos de mamão e por Santos et al. (2010), para o bioextrato de alho (Allium sativum L.), concentrações 500 a 5.000,00 mg/L, em Aspergillus niger. Na comparação com a testemunha sem tratamento todos os processos de obtenção dos bioextratos e todas as espécies vegetais apresentaram redução significativa no desenvolvimento do patógeno.

Tabela 2. Diâmetro médio de colônias de Phomopsis phaseoli var. sojae tratadas com extrato de alho, cebola, cipreste, gengibre, pinhão manso e tiririca obtidos na forma in natura e/ou secos submetidos ou não a autoclavagem

(Continua)

\begin{tabular}{|c|c|c|c|c|c|c|}
\hline \multirow[b]{2}{*}{ Processo } & \multicolumn{6}{|c|}{ Diâmetro médio das colônias aos 7 dias (cm) } \\
\hline & Alho & Cebola & Cipreste & Gengibre & $\begin{array}{l}\text { Pinhão } \\
\text { Manso }\end{array}$ & Tiririca \\
\hline Carbendazin + Tiran & $0,8 \mathrm{a}$ & 0,8 a & 0,8 a & $0,8 \mathrm{a}$ & 0,8 a & 0,8 a \\
\hline In natura autoclavado & $4,1 \mathrm{~b}$ & $6,9 \mathrm{~b}$ & $3,0 \mathrm{~b}$ & $4,9 \mathrm{~b}$ & $3,8 \mathrm{~b}$ & $5,8 \mathrm{~b}$ \\
\hline $\begin{array}{l}\text { In natura não } \\
\text { autoclavado }\end{array}$ & $1,2 \mathrm{~b}$ & $7,0 \mathrm{~b}$ & $3,0 \mathrm{~b}$ & $4,4 \mathrm{~b}$ & $3,6 \mathrm{~b}$ & $4,7 \mathrm{~b}$ \\
\hline
\end{tabular}


(Conclusão)

\begin{tabular}{|c|c|c|c|c|c|c|}
\hline \multirow[b]{2}{*}{ Processo } & \multicolumn{6}{|c|}{ Diâmetro médio das colônias aos 7 dias $(\mathrm{cm})$} \\
\hline & Alho & Cebola & Cipreste & Gengibre & $\begin{array}{l}\text { Pinhão } \\
\text { Manso }\end{array}$ & Tiririca \\
\hline Seco autoclavado & $4,1 \mathrm{~b}$ & $5,5 \mathrm{~b}$ & $3,6 \mathrm{~b}$ & $2,3 \mathrm{~b}$ & $4,7 \mathrm{~b}$ & $5,5 \mathrm{~b}$ \\
\hline Seco não autoclavado & $0,8 \mathrm{a}$ & $5,4 \mathrm{~b}$ & $3,6 \mathrm{~b}$ & $2,3 \mathrm{~b}$ & $5,6 \mathrm{~b}$ & $5,0 \mathrm{~b}$ \\
\hline Testemunha & $7,6 \mathrm{c}$ & $7,6 \mathrm{c}$ & $7,6 \mathrm{c}$ & $7,6 \mathrm{c}$ & $7,6 \mathrm{c}$ & $7,6 \mathrm{c}$ \\
\hline CV (\%) & 5,2 & 5,1 & 7,1 & 6,6 & 5,5 & 8,5 \\
\hline
\end{tabular}

Médias seguidas de mesma letra na coluna não diferem entre si pelo teste de Dunnett a $5 \%$ de probabilidade.

Nos resultados apresentados na Tabela 03 verifica-se que todos os bioextratos utilizados diferiram significativamente da testemunha com tratamento químico (Carbendazin + Tiran), sendo que os bioextratos de alho, pinhão manso e tiririca não interferiram no desenvolvimento de Fusarium spp. E, consequentemente, não diferiram da testemunha sem tratamento em nenhum dos processos utilizados. Os bioextratos de cebola in natura não autoclavado, cipreste submetido à secagem autoclavado, gengibre in natura autoclavado e seco não autoclavado apresentaram reduções significativas no desenvolvimento do patógeno, com relação à testemunha sem tratamento. Flávio et al. (2014) observaram que o bioextrato aquoso de canela, (Cinnamomum zeylanicum) concentração $10-30 \% \mathrm{v} / \mathrm{v}$, reduziu a presença do fungo Curvularia em sementes de sorgo.

Tabela 3. Diâmetro médio de colônias de Fusarium spp tratadas com extrato de alho, cebola, cipreste, gengibre, pinhão manso e tiririca obtidos na forma in natura e/ou secos submetidos ou não a autoclavagem

(Continua)

\begin{tabular}{|c|c|c|c|c|c|c|}
\hline \multirow[b]{2}{*}{ Processo } & \multicolumn{6}{|c|}{ Diâmetro médio das colônias aos 7 dias $(\mathrm{cm})$} \\
\hline & Alho & Cebola & Cipreste & Gengibre & $\begin{array}{l}\text { Pinhão } \\
\text { Manso }\end{array}$ & Tiririca \\
\hline $\begin{array}{l}\text { Carbendazin + } \\
\text { Tiran }\end{array}$ & $0,8 \mathrm{a}$ & $0,8 \mathrm{a}$ & $0,8 \mathrm{a}$ & $0,8 \mathrm{a}$ & $0,8 \mathrm{a}$ & $0,8 \mathrm{a}$ \\
\hline In natura autoclavado & $5,4 \mathrm{~b}$ & $4,3 \mathrm{c}$ & $3,9 \mathrm{c}$ & $3,3 \mathrm{~b}$ & $4,1 \mathrm{~b}$ & $4,9 \mathrm{~b}$ \\
\hline $\begin{array}{l}\text { In natura não } \\
\text { autoclavado }\end{array}$ & $4,0 \mathrm{~b}$ & $3,2 \mathrm{~b}$ & $3,8 \mathrm{c}$ & $3,5 \mathrm{c}$ & $4,7 \mathrm{~b}$ & $4,7 \mathrm{~b}$ \\
\hline
\end{tabular}


(Conclusão)

\begin{tabular}{lcccccc}
\hline \multirow{2}{*}{ Processo } & \multicolumn{5}{c}{ Diâmetro médio das colônias aos 7 dias (cm) } \\
\cline { 2 - 7 } & Alho & Cebola & Cipreste & Gengibre & $\begin{array}{c}\text { Pinhão } \\
\text { Manso }\end{array}$ & Tïririca \\
\hline Seco autoclavado & $4,2 \mathrm{~b}$ & $4,4 \mathrm{c}$ & $3,2 \mathrm{~b}$ & $3,6 \mathrm{c}$ & $5,1 \mathrm{~b}$ & $4,6 \mathrm{~b}$ \\
Seco não autoclavado & $4,2 \mathrm{~b}$ & $3,8 \mathrm{c}$ & $4,1 \mathrm{c}$ & $2,7 \mathrm{~b}$ & $5,7 \mathrm{~b}$ & $5,7 \mathrm{~b}$ \\
\hdashline Testemunha & $\mathbf{4 , 9} \mathrm{b}$ & $\mathbf{4 , 9} \mathrm{c}$ & $\mathbf{4 , 9} \mathrm{c}$ & $\mathbf{4 , 9} \mathrm{c}$ & $\mathbf{4 , 9} \mathrm{b}$ & $\mathbf{4 , 9} \mathbf{b}$ \\
\hline CV $(\%)$ & $\mathbf{1 8 , 2}$ & $\mathbf{1 8 , 4}$ & $\mathbf{1 8 , 6}$ & $\mathbf{2 3 , 8}$ & $\mathbf{1 6 , 2}$ & $\mathbf{1 3 , 5}$ \\
\hline
\end{tabular}

Médias seguidas de mesma letra na coluna não diferem entre si pelo teste de Dunnett a 5\% de probabilidade.

A percentagem de germinação das sementes de soja submetidas aos diferentes tratamentos é apresentada na Tabela 4, onde se pode observar que todos os bioextratos apresentaram índices de germinação ao tratamento com fungicida, porém os bioextratos de alho in natura autoclavado ou seco não autoclavado, e de gengibre in natura autoclavado ou seco autoclavado, apresentaram índices de germinação superiores à testemunha (sem tratamento). Estes resultados sugerem que é possível aumentar a porcentagem de germinação utilizando bioextratos vegetais no tratamento de sementes, como sugerem Flávio et al. 2014 para o bioextrato aquoso de canela (Cinnamomum zeylanicum) 10-30\% v/v no tratamento de sementes de sorgo. A contaminação das sementes por patógenos é responsável pela redução do índice de germinação das sementes, conforme reportado por Henning (2005), o qual destaca que lotes de sementes com altos índices de infecção de Phomopsis sp. apresentam baixo percentual de germinação.

Tabela 4. Percentagem de germinação de sementes de soja tratadas com diferentes extratos vegetais e fungicida

(Continua)

\begin{tabular}{lcccccc}
\hline & \multicolumn{6}{c}{ Germinação (\%) } \\
\cline { 2 - 7 } \multicolumn{1}{c}{ Processo } & Alho & Cebola & Cipreste & Gengibre & $\begin{array}{l}\text { Pinhão } \\
\text { Manso }\end{array}$ & Tiririca \\
\hline $\begin{array}{l}\text { Carbendazin }+ \\
\text { Tiran }\end{array}$ & $44,3 \mathrm{a}$ & $\mathbf{4 4 , 3} \mathrm{a}$ & $\mathbf{4 4 , 3} \mathrm{a}$ & $\mathbf{4 4 , 3} \mathrm{a}$ & $\mathbf{4 4 , 3} \mathrm{a}$ & $\mathbf{4 4 , 3} \mathrm{a}$ \\
\hdashline $\begin{array}{l}\text { In natura } \\
\text { autoclavado }\end{array}$ & $24,5 \mathrm{~b}$ & $17,5 \mathrm{~b}$ & $20,0 \mathrm{~b}$ & $23,3 \mathrm{~b}$ & $17,8 \mathrm{~b}$ & $14,3 \mathrm{~b}$ \\
& & & & & & \\
\hline
\end{tabular}


(Conclusão)

\begin{tabular}{|c|c|c|c|c|c|c|}
\hline \multirow[b]{2}{*}{ Processo } & \multicolumn{6}{|c|}{ Germinação (\%) } \\
\hline & Alho & Cebola & Cipreste & Gengibre & $\begin{array}{l}\text { Pinhão } \\
\text { Manso }\end{array}$ & Tiririca \\
\hline $\begin{array}{l}\text { In natura não } \\
\text { autoclavado }\end{array}$ & $16,8 \mathrm{c}$ & $20,5 \mathrm{~b}$ & $17,8 \mathrm{~b}$ & $15,8 \mathrm{c}$ & $11,3 \mathrm{~b}$ & $19,3 \mathrm{~b}$ \\
\hline Seco autoclavado & $19,0 \mathrm{c}$ & $20,3 \mathrm{~b}$ & $20,3 \mathrm{~b}$ & $23,0 \mathrm{~b}$ & $15,3 \mathrm{~b}$ & $26,0 \mathrm{~b}$ \\
\hline $\begin{array}{l}\text { Seco não } \\
\text { autoclavado }\end{array}$ & $23,8 \mathrm{~b}$ & $12,3 \mathrm{~b}$ & $16,5 \mathrm{~b}$ & $21,5 \mathrm{c}$ & $16,3 \mathrm{~b}$ & $16,3 \mathrm{~b}$ \\
\hline Testemunha & $15,3 \mathrm{c}$ & $15,3 \mathrm{~b}$ & $15,3 \mathrm{~b}$ & $15,3 \mathrm{c}$ & $15,3 \mathrm{~b}$ & $15,3 \mathrm{~b}$ \\
\hline CV (\%) & 8,5 & 11,4 & 7,6 & 9,3 & 20,7 & 13,4 \\
\hline
\end{tabular}

Médias seguidas de mesma letra na coluna não diferem entre si pelo teste de Dunnett a $5 \%$ de probabilidade.

O ajuste da dose e da forma de aplicação dos óleos essenciais e bioextratos de plantas na busca do equilíbrio entre o controle dos patógenos e a melhoria do índice de germinação tem sido objetivo de muitas pesquisas. Trabalhos realizados por Hellen et al. (2012), Medeiros et al. (2013a), Souza et al. (2010), Maia et al. (2011) e Flávio et al. (2012) indicam redução do percentual de germinação na medida em que obtinham controle dos patógenos. Outros autores como Flávio, et al. (2014) obtiveram melhorias da qualidade fisiológica das sementes, usando óleo essencial de alfavaca cravo (Ocimum gratissimum) e bioextrato de canela (Cinnamomum zeylanicum. Por outro lado, Medeiros et al. (2013a) observaram melhora na germinação das sementes de Clitoria fairchildiana Howard quando tratadas com bioextrato de melão-de-são-caetano 1.000,00 ppm (Momordica charantia).

Os resultados do teste de envelhecimento acelerado apresentados na Tabela 05 indicam que todos os bioextratos aplicados apresentaram percentual de plântulas normais inferiores à testemunha com tratamento com fungicida, diferindo significativamente desta. Os bioextratos de alho, cipreste e pinhão manso não diferiram da testemunha sem tratamento em todos os processos de elaboração dos bioextratos utilizados. O bioextrato de cebola quando utilizado na forma in natura não autoclavado apresentou melhora significativa do percentual de plântulas normais. Entretanto, quando utilizado na forma seca autoclavada esse bioextrato interferiu reduzindo substancialmente a germinação das sementes, particularmente 
quando comparada com a testemunha sem tratamento. Os bioextratos de gengibre in natura não autoclavado e tiririca obtido após secagem não autoclavada melhoraram significativamente o percentual de plântulas normais com relação à testemunha sem tratamento.

Tabela 5. Porcentagem de plântulas normais do teste de envelhecimento acelerado em sementes de soja após submetidas a diferentes tratamentos com estratos vegetais e fungicida

\begin{tabular}{|c|c|c|c|c|c|c|}
\hline \multirow[b]{2}{*}{ Processo } & \multicolumn{6}{|c|}{ Envelhecimento Acelerado (\%) } \\
\hline & Alho & Cebola & Cipreste & Gengibre & $\begin{array}{l}\text { Pinhão } \\
\text { Manso }\end{array}$ & Tiririca \\
\hline $\begin{array}{l}\text { Carbendazin + } \\
\text { Tiran }\end{array}$ & 37,8 a & 37,8 a & $37,8 \mathrm{a}$ & $37,8 \mathrm{a}$ & $37,8 \mathrm{a}$ & 37,8 a \\
\hline $\begin{array}{l}\text { In natura } \\
\text { autoclavado }\end{array}$ & $14,5 \mathrm{~b}$ & $12,0 \mathrm{c}$ & $12,3 \mathrm{~b}$ & $16,0 \mathrm{c}$ & $15,0 \mathrm{~b}$ & $11,0 \mathrm{c}$ \\
\hline $\begin{array}{l}\text { In natura não } \\
\text { autoclavado }\end{array}$ & $15,5 \mathrm{~b}$ & $18,3 \mathrm{~b}$ & $15,5 \mathrm{~b}$ & $19,0 \mathrm{~b}$ & $16,8 \mathrm{~b}$ & $11,0 \mathrm{c}$ \\
\hline Seco autoclavado & $15,8 \mathrm{~b}$ & $7,3 \mathrm{~d}$ & $12,5 \mathrm{~b}$ & $11,8 \mathrm{c}$ & $13,3 \mathrm{~b}$ & $15,0 \mathrm{c}$ \\
\hline $\begin{array}{l}\text { Seco não } \\
\text { autoclavado }\end{array}$ & $15,8 \mathrm{~b}$ & $10,0 \mathrm{c}$ & $13,0 \mathrm{~b}$ & $12,8 \mathrm{c}$ & $13,3 \mathrm{~b}$ & $20,0 \mathrm{~b}$ \\
\hline Testemunha & $12,0 \mathrm{~b}$ & $12,0 \mathrm{c}$ & $12,0 \mathrm{~b}$ & $12,0 \mathrm{c}$ & $12,0 \mathrm{~b}$ & $12,0 \mathrm{c}$ \\
\hline CV (\%) & 7,2 & 7,8 & 9,4 & 8,8 & 14,4 & 7,8 \\
\hline
\end{tabular}

Médias seguidas de mesma letra na coluna não diferem entre si pelo teste de Dunnett a $5 \%$ de probabilidade.

O tratamento com Derosal Plus ${ }^{\circledR}$ (Carbendazin + Tiran) aplicado nas sementes de soja reduziu a zero as contaminações de Fusarium spp. e Phomopsis phaseoli var. sojae (Tabelas 06 e 07), os quais apresentavam índices de contaminação de 14,3 e 46,5\%, respectivamente. O controle dos patógenos associados às sementes fez com que os índices de plântulas normais fossem aumentados em 29 e 25,8\%, (Tabelas 04 e 05) respectivamente, para os testes de germinação e de envelhecimento acelerado.

Os dados das Tabelas 06 e 07 mostram que todos os bioextratos utilizados não apresentaram eficiência na redução da contaminação das sementes por Fusarium spp. e Phomopsis phaseoli var. sojae, pois todos os tratamentos não diferiram da 
testemunha sem tratamento. Apesar das melhorias no percentual de germinação e de envelhecimento acelerado, apontado por alguns bioextratos, o percentual de contaminação das sementes não retratou redução; a justificativa mais provável para este fato deve-se ao teste de sanidade, pois este avalia somente a presença ou ausência do patógeno na semente, não quantificando a infecção apresentada.

Tabela 6. Percentagem de sementes de soja contaminadas com Fusarium spp. após submetidas a diferentes tratamentos com extratos vegetais e fungicida

Percentual de sementes contaminadas (\%)

\begin{tabular}{|c|c|c|c|c|c|c|}
\hline Processo & Alho & Cebola & Cipreste & Gengibre & $\begin{array}{l}\text { Pinhão } \\
\text { Manso }\end{array}$ & Tiririca \\
\hline $\begin{array}{l}\text { Carbendazin }+ \\
\text { Tiran }\end{array}$ & $0,0 \mathrm{a}$ & $0,0 \mathrm{a}$ & $0,0 \mathrm{a}$ & $0,0 \mathrm{a}$ & $0,0 \mathrm{a}$ & $0,0 \mathrm{a}$ \\
\hline $\begin{array}{l}\text { In natura } \\
\text { autoclavado }\end{array}$ & $13,5 \mathrm{~b}$ & $12,3 \mathrm{~b}$ & $12,8 \mathrm{~b}$ & $12,3 \mathrm{~b}$ & $13,8 \mathrm{~b}$ & $12,8 \mathrm{~b}$ \\
\hline $\begin{array}{l}\text { In natura não } \\
\text { autoclavado }\end{array}$ & $11,8 \mathrm{~b}$ & $13,0 \mathrm{~b}$ & $13,0 \mathrm{~b}$ & $13,5 \mathrm{~b}$ & $11,5 \mathrm{~b}$ & $12,3 \mathrm{~b}$ \\
\hline Seco autoclavado & $11,3 \mathrm{~b}$ & $12,3 \mathrm{~b}$ & $12,0 \mathrm{~b}$ & $10,8 \mathrm{~b}$ & $12,3 \mathrm{~b}$ & $12,5 \mathrm{~b}$ \\
\hline $\begin{array}{l}\text { Seco não } \\
\text { autoclavado }\end{array}$ & $13,5 \mathrm{~b}$ & $12,5 \mathrm{~b}$ & $14,0 \mathrm{~b}$ & $14,3 \mathrm{~b}$ & $12,8 \mathrm{~b}$ & $11,0 \mathrm{~b}$ \\
\hline Testemunha & $14,3 \mathrm{~b}$ & $14,3 \mathrm{~b}$ & $14,3 \mathrm{~b}$ & $14,3 \mathrm{~b}$ & $14,3 \mathrm{~b}$ & $14,3 \mathrm{~b}$ \\
\hline CV (\%) & 26,9 & 26,5 & 26,4 & 28,5 & 23,9 & 22,6 \\
\hline
\end{tabular}

Médias seguidas de mesma letra na coluna não diferem entre si pelo teste de Dunnett a $5 \%$ de probabilidade.

Tabela 7. Percentagem de sementes de soja contaminadas com Phomopsis phaseoli var. sojae tratadas com diferentes extratos vegetais e fungicida (Continua)

Percentual de sementes contaminadas (\%)

\begin{tabular}{|c|c|c|c|c|c|c|}
\hline Processo & Alho & Cebola & Cipreste & Gengibre & $\begin{array}{l}\text { Pinhão } \\
\text { Manso }\end{array}$ & Tiririca \\
\hline Carbendazin + Tiran & $0,0 \mathrm{a}$ & $0,0 \mathrm{a}$ & $0,0 \mathrm{a}$ & $0,0 \mathrm{a}$ & $0,0 \mathrm{a}$ & $0,0 \mathrm{a}$ \\
\hline In natura autoclavado & $48,3 \mathrm{~b}$ & $46,0 \mathrm{~b}$ & $46,0 \mathrm{~b}$ & $44,8 \mathrm{~b}$ & $44,0 \mathrm{~b}$ & $43,3 \mathrm{~b}$ \\
\hline $\begin{array}{l}\text { In natura não } \\
\text { autoclavado }\end{array}$ & $49,5 \mathrm{~b}$ & $41,8 \mathrm{~b}$ & $47,0 \mathrm{~b}$ & $50,8 \mathrm{~b}$ & $45,3 \mathrm{~b}$ & $47,8 \mathrm{~b}$ \\
\hline
\end{tabular}


(Conclusão)

\begin{tabular}{lcccccc}
\hline \multirow{2}{*}{\multicolumn{1}{c}{ Processo }} & \multicolumn{5}{c}{ Percentual de sementes contaminadas (\%) } \\
\cline { 2 - 7 } & Alho & Cebola & Cipreste & Gengibre & $\begin{array}{l}\text { Pinhão } \\
\text { Manso }\end{array}$ & Tiririca \\
\hline Seco autoclavado & $47,5 \mathrm{~b}$ & $45,8 \mathrm{~b}$ & $48,5 \mathrm{~b}$ & $46,8 \mathrm{~b}$ & $49,5 \mathrm{~b}$ & $49,5 \mathrm{~b}$ \\
Seco não autoclavado & $44,3 \mathrm{~b}$ & $47,3 \mathrm{~b}$ & $44,5 \mathrm{~b}$ & $48,0 \mathrm{~b}$ & $43,3 \mathrm{~b}$ & $46,8 \mathrm{~b}$ \\
\hdashline Testemunha & $\mathbf{4 6 , 5} \mathbf{b}$ & $\mathbf{4 6 , 5} \mathbf{b}$ & $\mathbf{4 6 , 5} \mathbf{b}$ & $\mathbf{4 6 , 5} \mathbf{b}$ & $\mathbf{4 6 , 5} \mathbf{b}$ & $\mathbf{4 6 , 5} \mathbf{~ b}$ \\
\hline CV (\%) & $\mathbf{9 , 7}$ & $\mathbf{1 0 , 3}$ & $\mathbf{7 , 2}$ & $\mathbf{8 , 2}$ & $\mathbf{7 , 8}$ & $\mathbf{9 , 7}$ \\
\hline
\end{tabular}

Médias seguidas de mesma letra na coluna não diferem entre si pelo teste de Dunnett a $5 \%$ de probabilidade.

\section{CONCLUSÃO}

Nas condições de realização deste estudo conclui-se que, o bioextrato de alho seco não autoclavado controla eficientemente o desenvolvimento de Phomopsis phaseoli var. sojae in vitro. Entretanto, essa capacidade fungitóxica do bioextrato de alho é perdida quando submetido à autoclavagem. Por outro lado, bioextrato de cebola seco autoclavado reduz o vigor de sementes de soja. Os bioextratos obtidos com a matéria seca de alho, cebola e gengibre foram mais eficientes no controle in vitro de $P$. phaseoli var. sojae, e o de tiririca reduziu o índice de plântulas anormais. Além disso, a secagem reduziu a eficiência dos bioextratos de cipreste e pinhão manso.

\section{AGRADECIMENTOS}

Os autores agradecem a Fundação de Amparo à Pesquisa do Estado de Mato Grosso (Fapemat- Processo número 336236/2012), pelo apoio financeiro e a Universidade do Estado de Mato Grosso (UNEMAT), pela estrutura física para o desenvolvimento da pesquisa. 


\section{REFERÊNCIAS}

ANDRADE, M.A. CARDOSO, M.G.; BATISTA, L.R.; MALLET, A.C.T.; MACHADO, S.M.F. Óleos essenciais de Cymbopogon nardus, Cinnamomum zeylanicum e Zingiber officinale: composição, atividades antioxidante e antibacteriana. Revista Ciência Agronômica, v. 43, n. 2, p. 399-408, 2012.

AQUINO, C.F.; CATÃO, H.C.R.M.; SOARES, E.P.S.; MOURA, R.F.B.; SILVA, H.P.; SALES, N.L.P. Qualidade sanitária e fisiológica de sementes de girassol tratadas com hidrolatas e extratos de plantas. In: CONGRESSO BRASILEIRO DE MAMONA, 4 E SIMPÓSIO INTERNACIONAL DE OLEAGINOSAS ENERGÉTICAS, 1., 2010, João Pessoa. Anais João Pessoa: Embrapa Algodão, 2010. p.2159-2164.

AVILA, M. R, BACCINI, A. L, Avaliação da qualidade sanitária de sementes de soja no armazenamento, em função do tratamento com produto químico e extratos naturais. Informativo Abrates, v.11, n.2, p.158, 2001 Resumos.

AZEVEDO, L.F.; PASCUALI, L.C. Efeito inibitório de extratos vegetais hidroalcoólicos (metanol) "in vitro" em Fusarium spp. e na qualidade fisiológica de sementes de soja (Glycine max, L.). In: CONGRESSO INTERNO DE INICIAÇÃO CIENTIFICA, 4., e JORNADA CIENTIFICA DA UNEMAT, 12., Cáceres, 2008. Resumos... Cáceres. p. 5.

BRASIL. Ministério da Agricultura, Pecuária e Abastecimento. Secretaria de Defesa Agropecuária. Regras para análise de sementes. Ministério da Agricultura, Pecuária e Abastecimento. Brasília: Mapa/ACS, 2009. 399 p.

BUENO JÚNIOR, C.; CASTANHA, R. F.; IOST, R.; JULIATTI, F. C.; MORAIS, L. A. S. Extrato vegetal, fungicida e tempo de armazenamento dos produtos, no tratamento in vitro de sementes de feijoeiro contra Sclerotinia sclerotiorum. Ensaios e Ciência Ciências Biológicas, Agrárias e da Saúde, v. 16, n. 5, 2012.

CATÃO, H.C.R.M.; AQUINO, C.F.; SOARES, E.P.S.; MOURA, R.F.B.; SILVA, H.P.; SALES, N.L.P. Influência de extratos e hidrolatos de plantas na qualidade sanitária e fisiológica em sementes de mamona. In: CONGRESSO BRASILEIRO DE MAMONA, 
4 E SIMPÓSIO INTERNACIONAL DE OLEAGINOSAS ENERGÉTICAS, 1., 2010, João Pessoa. Anais ... João Pessoa: Embrapa Algodão, 2010. p. 2119-2123.

COWAN, M.M. Plant products as antimicrobial agents. Clinical Microbiology Reviews, v. 12, n. 4, p. 564-582, 1999

CRUZ, M. E. S.; SCHWAN-ESTRADA, K.R.F.; INGUE, M.H.; ÁVILA, M.R.; BATISTA, M.A.; STANGARLIN, J.R. Plantas medicinais e aromáticas promissoras no controle de patógenos de sementes de soja. In: CONGRESSO BRASILEIRO DE SOJA, 1., Londrina. 1999a. Anais... Londrina: Embrapa CNPSo, 1999a. 459p.

CRUZ, M. E. S.; SCHWAN-ESTRADA, K.R.F.; INGUE, M.H.; ÁVILA, M.R.; BATISTA, M.A.; STANGARLIN, J.R. Potencial de plantas medicinais no controle de patógenos que incidem sobre sementes de soja. In: CONGRESSO BRASILEIRO DE SOJA, 1., Londrina. 1999b. Anais... Londrina: Embrapa CNPSo, 1999b. 459p.

FALKENBERG, M.B.; SANTOS, R.I.; SIMÕES, C.M.O. FARMACOGNOSIA da planta ao medicamento. 2. ed. Porto Alegre: UFRGS; Florianópolis UFSC, 2000. 821p. Cap. 10: Introdução à análise Fitoquímica, p. 163-179.

FLÁVIO; N. S. D.S.; SALES; N. L. P. L.; AQUINO; F. S.; COSTA; C. A.; SOARES, E. P. S. Extratos aquosos de Cinnamomum zeylanicum e Mentha spicata no tratamento de sementes de sorgo. Horticultura Brasileira, v. 30, n. 2, p. S6333-S6339, 2012.

FLÁVIO; N.S.D. S.; SALES; N. L. P.; AQUINO; C. F.; SOARES; E. P. S.; AQUINO; L. F. S.; CATÃO, H.CÉSAR R.M. Qualidade sanitária e fisiológica de sementes de sorgo tratadas com extratos aquosos e óleos essenciais. Semina: Ciências Agrárias, v. 35, n. 1, p. $7-20,2014$.

FRIAS, D. F. R.; KOZUSNY-ANDREANI, D. I. Utilização de extratos de plantas medicinais e óleo e Eucaliptus no controle in vitro de Microsporum canis. Revista Cubana de Plantas Medicinales, v. 15, n.3, p.119-125, 2010.

GODOY; C.V.; ALMEIDA; Á. M. R.; SOARES; R. M.; SEIXAS; C. D. S.; DIAS; W. P.; MEYER; M. C.; COSTAMILAN; L. M.; HENNING, A. A. Doenças da soja (Glycine $\max (\mathrm{L}$.$) Merrill). [s.1.]: Sociedade Brasileira de Fitopatologia (SBF), 2014$. 
GOUVEA, A.; ZANOTTI, J.; LUCKMANN, D.; PIZZATTO, M.; MAZARO, S.M.; POSSENTI, J.C. Efeito de extratos vegetais em soja sob condições de laboratório e campo. Revista Brasileira de Agroecologia, v.6, n.2, p.70-78, 2011.

HENNING, A.A. Patologia e tratamento de sementes: noções gerais. Londrina: EMBRAPA - CNPSo, 2005. 52p. (EMBRAPA - CNPSo, documentos 264).

HILLEN, T.; SCHWAN-ESTRADA, K.R.F.; MESQUINI, R.M.; CRUZ, M.E.S.; STANGARLIN, J.R.; NOZAKI, M. Atividade antimicrobiana de óleos essenciais no controle de alguns fitopatógenos fúngicos in vitro e no tratamento de sementes. Revista Brasileira de Plantas Medicinais, v. 14, n. 3, p. 439-445, 2012.

INÁCIO, M. M.; PASCUALI, L. C.; ZELA, S. P.; PAULA, P. R. Diagnóstico de óleos essenciais, sobre o desenvolvimento de Phomopsis phaseoli var. sojae , Fusarium sp. e Macrophomina phaseolina. In: CONGRESSO INTERNO DE INICIAÇÃO CIENTIFICA, 5., e JORNADA CIENTIFICA DA UNEMAT, 2., Barra do Bugres, 2009. Resumos... Barra do Bugres. p. 5.

INQUE, M.H.; CRUZ, M.E.S.; SCHWAN-ESTRADA, K.R.F. Eficácia de plantas medicinais no controle de fungos fitopatogênicos que incidem sobre sementes de soja. Ponta Grossa, $5^{\circ}$ Simpósio Brasileiro de Patologia de Sementes. v. 1, p. 33. 1998. (Resumos)

KUMAR, K. P. S.; BHOWMIK, D; CHIRANJIB; BISWAJIT; TIWARI, P. Allium cepa: A Traditional medicinal herb and its health benefits. Journal of Chemical and Pharmaceutical Research, v. 2, n. 1, p. 283-291, 2010.

LEAL, A.C.K., AGRA, M.F. Estudo Farmacobotânico Comparativo das folhas de Jatropha molissima (Pohl) Baill.e Jatropha ribifolia (Pohl) Baill. (Euphorbiaceae). Acta Farmacéutica Bonaerense, v. 24, n. 1, p. 5-13, 2005.

MAIA, J.T.L.S.; BONFIM, F.P.G.; BARBOSA, C.K.R.; GUILHERME, D.O.; HONÓRIO, I.C.G.; MARTINS,E.R. Influência alelopática de hortelã (Mentha x villosa Huds.) sobre emergência de plântulas de alface (Lactuca sativa L.). Revista Brasileira de Plantas Medicinais, v. 13, n. 3, p. 253-257, 2011. 
MARCOS FILHO, J. Teste de envelhecimento acelerado. In: KRZYZANOWSKI, F.C.; VIEIRA, R.D.; FRANÇA NETO, J.B. (Ed.) Vigor de sementes: conceitos e testes. Londrina: ABRATES, 1999. p. 3.1; 3.24.

MEDEIROS, J. G. F.; ARAUJO NETO, A. C.; MENEZES, N. P.; NASCIMENTO, L. C. Sanidade e germinação de sementes de Clitoria fairchildiana tratadas com extratos de plantas. Pesquisa Florestal Brasileira, v. 33, n. 76, p. 403-408, 2013 a.

MEDEIROS, J.G. F.; ARAÚJO NETO, A. C.; MEDEIROS, D. S.; NASCIMENTO, L. C.;EDNA ALVES, U. Extratos Vegetais no Controle de Patógenos em Sementes de Pterogyne nitens Tul. Floresta e Ambiente; v. 20, n. 3, p. 384-390, 2013 b.

NASCIMENTO, L.C., NERY, A. R.; RODRIGUES, L.N. Controle de Colletotrichum gloeosporioidesem mamoeiro, utilizando em mamoeiro, utilizando extratos vegetais, indutores de resistência e fungicida. Acta Scientiarum. Agronomy, Maringá, v. 30, n. 3, p. 313-319, 2008.

NUUTILA M., PUUPPONEN-PIMIÃ R., AARNI M., OKSMAN-CALDENTEY K. M. Comparison of antioxidant activities of onion and garlic extracts by inhibition of lipid peroxidation and radical scavenging activity. Food Chemistry, v. 81, n. 4, p. 485-93, 2003.

PASTRO, D.C.; PASCUALI, L.C.; SANDRI, D.O.; ZELA, S.P.; SILVA, F.S. Diagnóstico de extratos vegetais com potencial para o controle fúngico, Enciclopédia Biosfera, v.8, n.14, p. 389-396, 2012.

PAULA, P.R.; PASCUALI, L.C.; ZELA, S.P.; INÁCIO, M.. Diagnóstico de extratos vegetais por extração hidroetanólica para o controle "in vitro" de Phomopsis phaseoli var. sojae e influência na qualidade de sementes de soja. In: CONGRESSO INTERNO DE INICIAÇÃO CIENTIFICA, 5., e JORNADA CIENTIFICA DA UNEMAT, 2., Barra do Bugres, 2009. Resumos... Barra do Bugres. p. 5.

RODRIGUES, E.A.; SCHWAN-ESTRADA, K.R.F.; STANGARLIN, J.R.; SCAPIM, C.A.; FIORI-TUTIDA, A.C.G. Potencial da planta medicinal Ocimum grassimum no controle de Bipolaris sorokiniana em sementes de trigo. Acta Scientiarum Agronomy, Maringá, v. 28, n. 2, p. 213-220, 2006. 
SAS. 1999. Statistical Analysis Software, version 9.1 System for Windows. SAS Institute Inc., Carry, NC, USA.

SANTOS, M.B.; SANTOS, C.Y.; ALMEIDA, M.A.; SANTOS, C.R.S.; SANT'ANNA, H.L.S.; SANTOS, O.S.N.; SILVA, F.; MARTINS, G.N. Efeito inibitório in vitro de extrato vegetal de Allium sativum sobre Aspergillus Níger. Tiegh. Rev. bras. plantas med. [online], Botucatu, v. 12, n. 1, p. 13-17, 2010.

SANTOS, P. L.; PRANDO, M. B.; MORANDO, R.; PEREIRA, G. V. N.; KRONKA, A. Z. Utilização de extratos vegetais em proteção de plantas. Enciclopédia Biosfera, v. 9 , n. 17 , p. 2562-2576, 2013.

SOUZA, P. F.; SILVA, G.H.; HENRIQUES, Í.G.N.; CAMPELO, G. J.; ALVES, G. S. Atividade antifúngica de diferentes concentrações de extrato de alho em sementes de ingá (Inga edulis). Revista Verde, v. 5, n. 5, p. 8-13, 2010.

SOUZA, V. M.; CARDOSO, S. B. Efeito alelopático do extrato de folhas de eucalyptus grandis sobre a germinação de lactuca sativa 1. ( alface) e phaseolus vulgaris 1.(feijão). Revista Eletrônica de Educação e Ciência, v. 3 n. 2, 2013.

VENTUROSO, L.R. Extratos vegetais no controle de fungos fitopatogênicos à soja. 2009. 99f. Dissertação (Mestrado em Produção Vegetal) - Universidade Federal da Grande Dourados, Dourados, 2009.

Recebido em: 31/07/2015 Aceito em: 31/03/2017 